\title{
Investigation on electronic transport property of cerium nitride nanoribbon-based molecular device: a first-principles study
}

\author{
R. Chandiramouli $\cdot$ P. Gopinath $\cdot$ S. Sriram
}

Received: 26 February 2014/ Accepted: 6 May 2014/Published online: 16 May 2014

(c) The Author(s) 2014. This article is published with open access at Springerlink.com

\begin{abstract}
Cerium nitride-based molecular device transport properties are investigated using density functional theory. The electronic transport properties are related in terms of density of states (DOS) and transmission spectrum. The peak maximum in the DOS arises due to the overlapping of different orbitals of cerium and nitrogen atoms. Under zero bias condition, the contribution of $f$ orbitals in cerium atom is seen whereas increasing the bias voltage, $f$ electrons gets perturbed and there is no contribution of $f$ orbital electrons for higher bias voltages. The electron density is seen more in nitrogen sites. The transmission of charges under various bias voltages gives the transmission spectrum. The geometry of structure and overlapping of orbitals leads to the variation in peak maximum in the nanoribbon. The electronic transport property of $\mathrm{CeN}$ nanoribbon provides an insight to enhance the transport property in functional nanomaterials.
\end{abstract}

Keywords Nanoribbon - Transport property - Density of states $\cdot$ Cerium nitride

\section{Introduction}

The rare-earth nitrides are hard and brittle materials, sensitive to hydrolysis and oxidation. These compounds crystallize in rocksalt type of structure. The potential application of rare-earth nitride is in spintronics [1]. Cerium nitride $(\mathrm{CeN})$ is one of the members of rare-earth nitrides that have unusual properties. Usually in rare-earth nitrides, trivalence dominates whereas in

R. Chandiramouli $(\bowtie) \cdot$ P. Gopinath $\cdot$ S. Sriram

School of Electrical and Electronics Engineering, SASTRA

University, Tirumalaisamudram 613 401, Thanjavur, India

e-mail: rcmoulii@gmail.com
$\mathrm{CeN}$, tetravalent dominates due to electronic configuration of $\mathrm{Ce}$ and small lattice parameter of $\mathrm{CeN}$. The band gap of $\mathrm{CeN}$ was found to be around $1.8 \mathrm{eV}, \mathrm{CeN}$ is analogous to the collapsed $\alpha$-phase of cerium metal where the $f$ electrons of cerium are peripatetic resembles the metallic property and cohesive property [2]. CeN can be synthesized by different methods such as radio frequency ion plating [3] and reacting Ce chips with $\mathrm{NH}_{3}$ gas [4]. Xiao et al. [5] reported thickness-dependent valence fluctuation on CeN thin films. Modifying the materials' properties finds its potential importance for technological applications to develop functional materials in nanoelectronics. The prime focus is to characterize and manipulate the quantum states of the molecule. Molecular electronics uses the molecules having a dimension below $100 \mathrm{~nm}$ in the form of nanoribbon, nanowire or in nanosheet form as a substitute for the solid-state semiconductor devices. Molecular electronics find the use of single molecule or group of molecules in nanoregime to function as basic element in electronic devices. Molecular electronic devices mainly use graphene, which is a promising candidate for nanoelectronics, photonic devices, nanosensors and nanomechanical devices. With this as motivation, a literature survey was conducted and it was found that not much work has been reported on CeN-based molecular device. Density functional theory (DFT) is an efficient method to study the structural and electronic transport properties of $\mathrm{CeN}$ nanoribbon. In the present work, $\mathrm{CeN}$ nanoribbon is placed between the electrodes and the electronic transport properties of CeN nanoribbon are studied using DFT and the results are reported.

\section{Methods}

The investigation of $\mathrm{CeN}$ nanoribbon is carried out through density functional theory (DFT) using 
Electrode Up

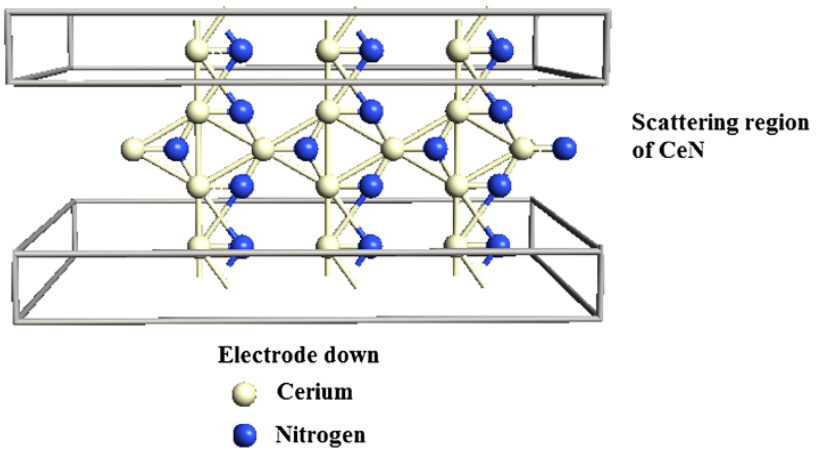

Fig. 1 Schematic diagram of the CeN nanoribbon with electrodes
TranSIESTA module in SIESTA package [6]. DFT method is a good approach to study the electronic transport property of nanomaterials. TranSIESTA enables the molecular electronic device based on DFT to estimate the self-consistent electronic structure of nanomaterials within the three-dimensional electrodes at different chemical potentials. DFT methods explore the information concerning the scattering region, transmission coefficient, electron current and non-equilibrium forces in the system [7-11]. In the present work the calculation was based on DFT with the electronic exchange, and correlation effects are designated by generalized gradient approximation (GGA). The
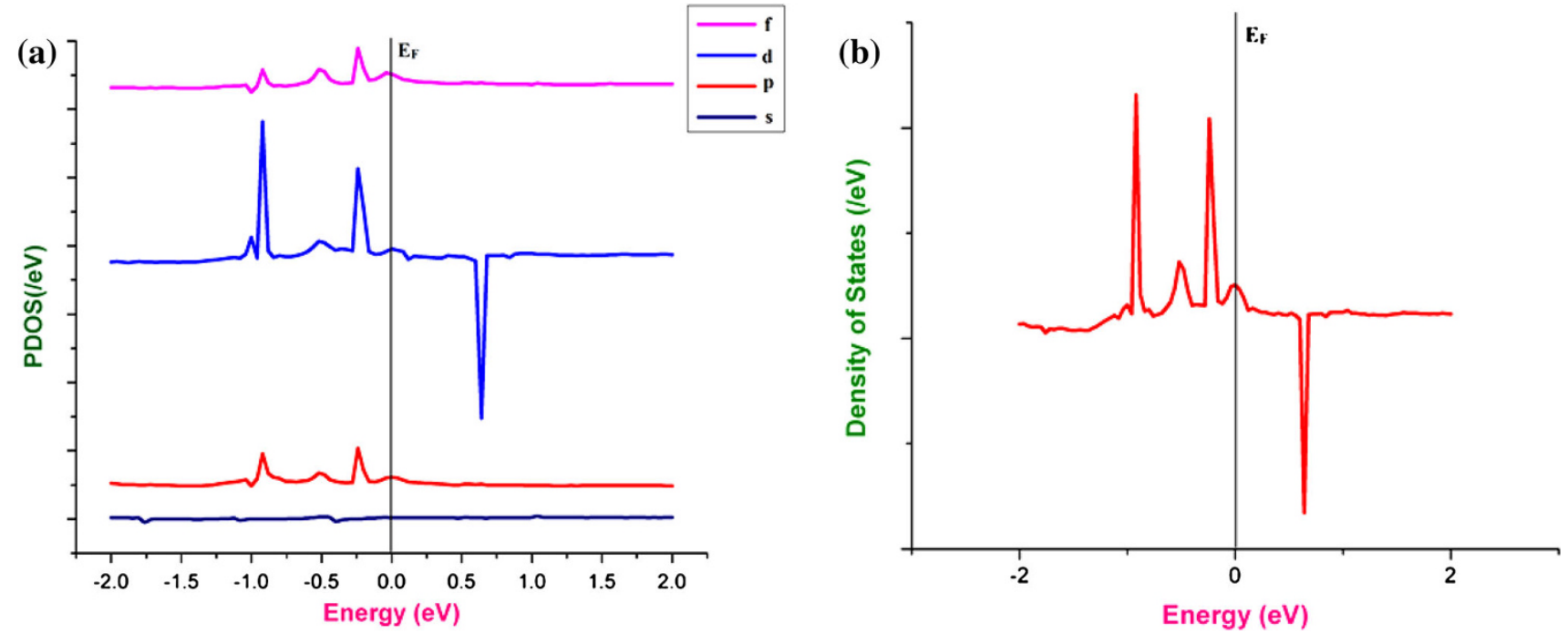

Fig. 2 a PDOS of CeN nanoribbon at $0 \mathrm{~V}$ bias. b DDOS of CeN nanoribbon at $0 \mathrm{~V}$ bias
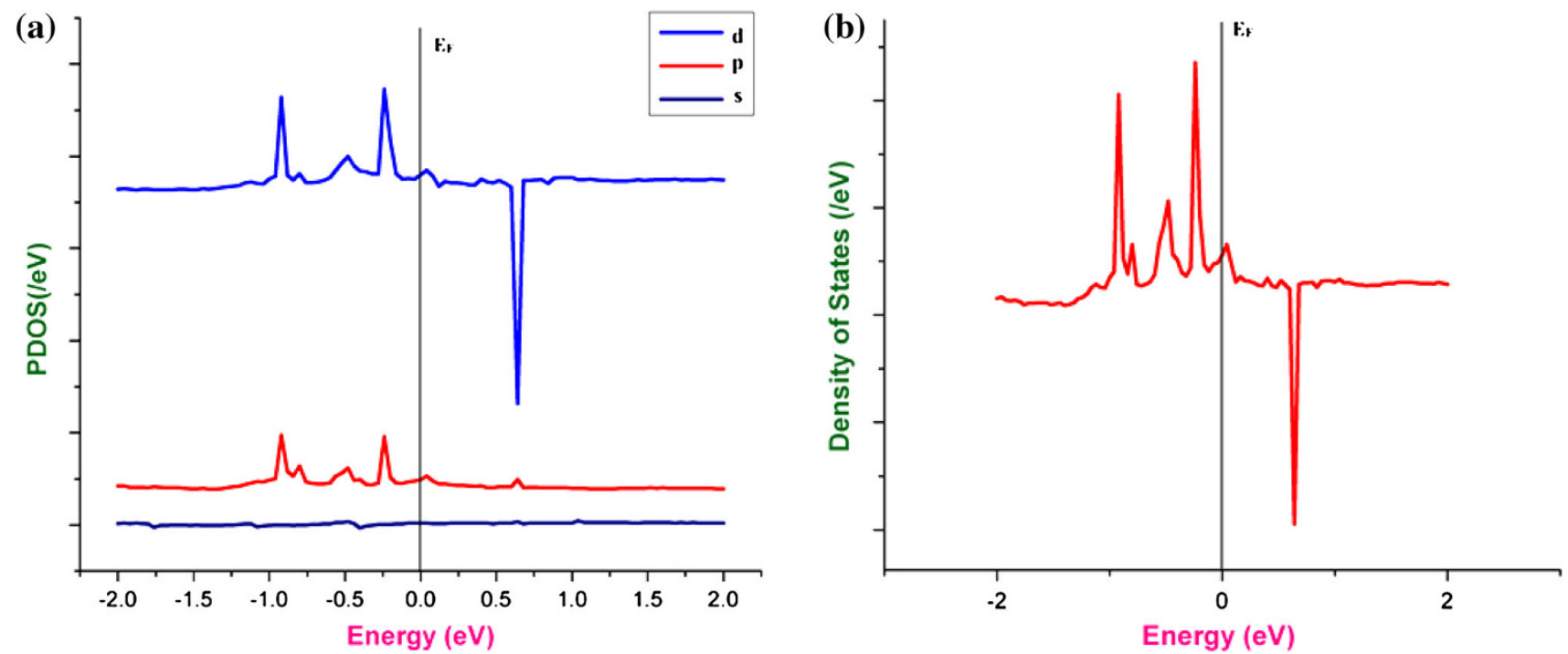

Fig. 3 a PDOS of CeN nanoribbon at $0.5 \mathrm{~V}$ bias. b DDOS of $\mathrm{CeN}$ nanoribbon at $0.5 \mathrm{~V}$ bias 
electron-electron interactions of the molecules are estimated with GGA through Perdew-Burke-Ernzerhof (PBE) exchange correlation $[12,13]$. In the present work, GGA-PPE functional is used throughout the calculation [14-17]. The optimization of the molecular geometry is taken into picture by decreasing the atomic forces of the atoms, which is smaller than $0.05 \mathrm{eV} / \AA$. The sampling of the Brillouin zones is done with $3 \times 3 \times 50 \mathrm{k}$ points. The real space grids for the electrostatic potentials are calculated with the mesh cut-off energy of $10^{-5} \mathrm{eV}$. The atomic coordinates are adjusted without any symmetry constraint with a convergence criteria of $0.05 \mathrm{eV}$ for energy and $0.05 \AA$ for displacement. CeN nanoribbon electronic transport property is calculated using vacuum padding of $10 \AA$, which is modeled along $\mathrm{x}$ - and $\mathrm{y}$-axis to avoid the interaction of the nanoribbon with their periodic images. The atoms in nanoribbon are free to move in their positions until a convergence criteria with the force less than $0.05 \mathrm{eV} / \AA$ on each and every atom in the $\mathrm{CeN}$ nanoribbon is achieved. The electronic transport system is being divided into three subsystems: left electrode, right electrode, and the scattering region which consists of $\mathrm{CeN}$. The electronic transport properties and optimization of the $\mathrm{CeN}$ scattering region are carried out by the double zeta plus polarization (DZP) basis set for the
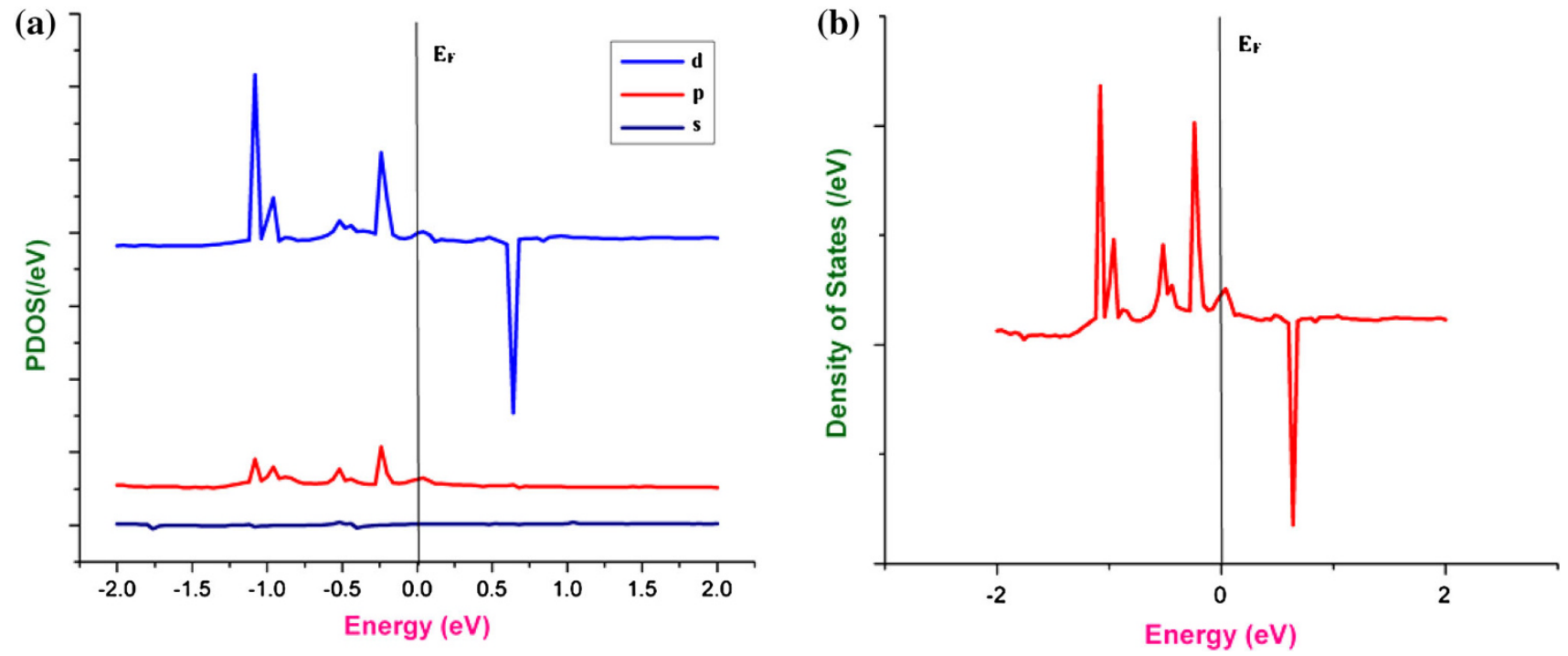

Fig. 4 a PDOS of CeN nanoribbon at $1 \mathrm{~V}$ bias. b DDOS of CeN nanoribbon at $1 \mathrm{~V}$ bias
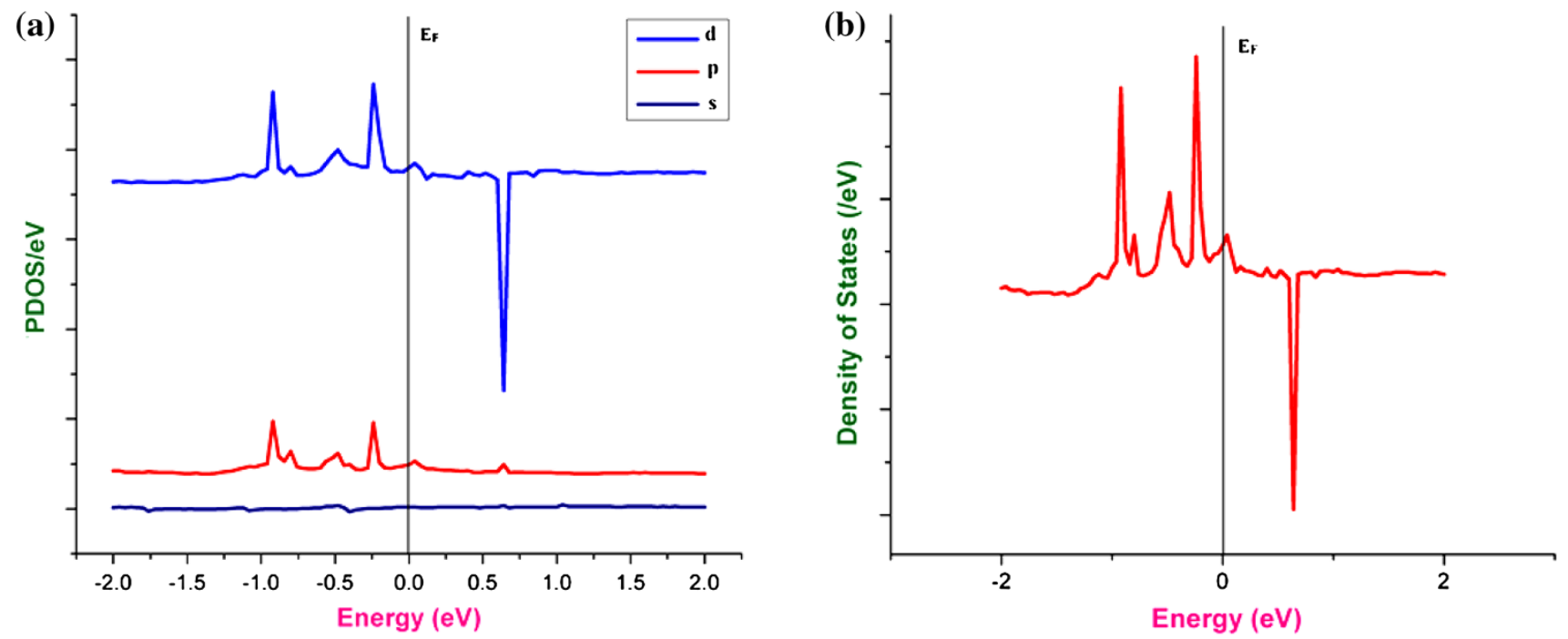

Fig. 5 a PDOS of CeN nanoribbon at $1.5 \mathrm{~V}$ bias. b DDOS of $\mathrm{CeN}$ nanoribbon at $1.5 \mathrm{~V}$ bias 
CeN nanoribbon scattering region and for electrode region in the present study.

\section{Results and discussion}

\section{Structure of CeN nanoribbon}

The structure of nanoribbon is chosen from the International Centre for Diffraction Data (ICDD card number: 89-5220), based on the structure, CeN nanoribbon was designed with hexagonal layers attached to the electrodes. The adjacent position of nanoribbon has the cerium and nitrogen atoms, which are attached to the electrode up and electrode down. CeN nanoribbon consists of 16 cerium atoms and 16 nitrogen atoms. The scattering region consists of ten cerium atoms and ten nitrogen atoms. The up and down electrodes are attached to three cerium and three nitrogen atoms. The potential difference is maintained between the up electrode and down electrode. The up electrode potential is varied between $0,0.5,1$ and $1.5 \mathrm{~V}$, whereas the down electrode is kept at ground potential. $\mathrm{CeN}$ act as the scattering region, the density of states (DOS) and transmission spectrum of $\mathrm{CeN}$ scattering region are studied and reported. The schematic diagram of $\mathrm{CeN}$ nanoribbon with the electrodes is illustrated in the Fig. 1.

\section{Density of states of CeN nanoribbon}

The DOS spectrum of the scattering region gives the perception to the presence of charge in particular interval of energy with the applied bias voltage [18-21]. In the present work, the voltage is varied within the interval of $0-1.5 \mathrm{~V}$ across the CeN nanoribbon in terms of $0.5 \mathrm{~V}$. The bias window is set between $[-\mathrm{V} / 2, \mathrm{~V} / 2]$, hence the Fermi level $\left(E_{\mathrm{F}}\right)$ is taken as zero. The contribution due to the different orbitals gives rise to projected density of states (PDOS), the device density of states (DDOS) will depend upon the PDOS of the molecules present in the scattering region. The electronic configuration of cerium is $[\mathrm{Xe}] 4 f^{1} 5 d^{1} \quad 6 s^{2}$ and nitrogen is $1 s^{2} 2 s^{2} 2 p^{3}$, and the overlapping of different orbitals leads to the DOS of PDOS at different energy interval. The superposition of $s, p, d, f$ orbitals of cerium and nitrogen leads to the device DOS. Figure $2 \mathrm{a}$ and $\mathrm{b}$ represents the PDOS and DDOS of CeN nanoribbon at zero bias voltage. In the case of zero bias condition, a peak maximum is seen at -0.2 and $-0.8 \mathrm{eV}$ in $p, d, f$ orbitals due to the overlapping of the $p, d, f$ orbitals of cerium with $s$ and $p$ orbitals of nitrogen, a decrease in DOS is observed at $p$ orbital at $0.6 \mathrm{eV}$ in the conduction band. In DDOS spectrum for zero bias, more peaks are observed at the negative energy which represents the valence band of $\mathrm{CeN}$ nanoribbon, it implies that more number of states is localized near

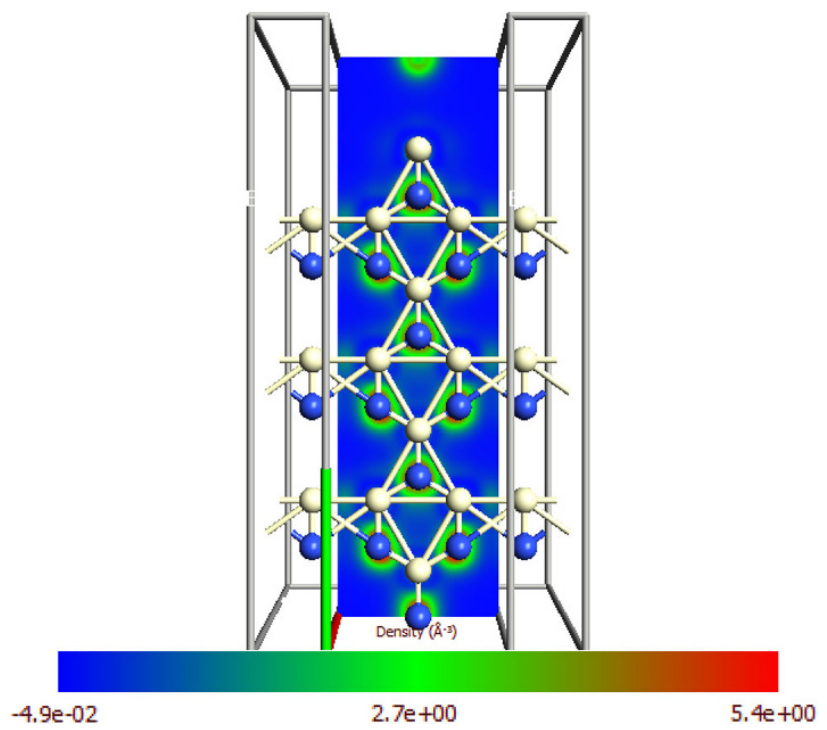

Fig. 6 Electron density in $\mathrm{CeN}$ nanoribbon

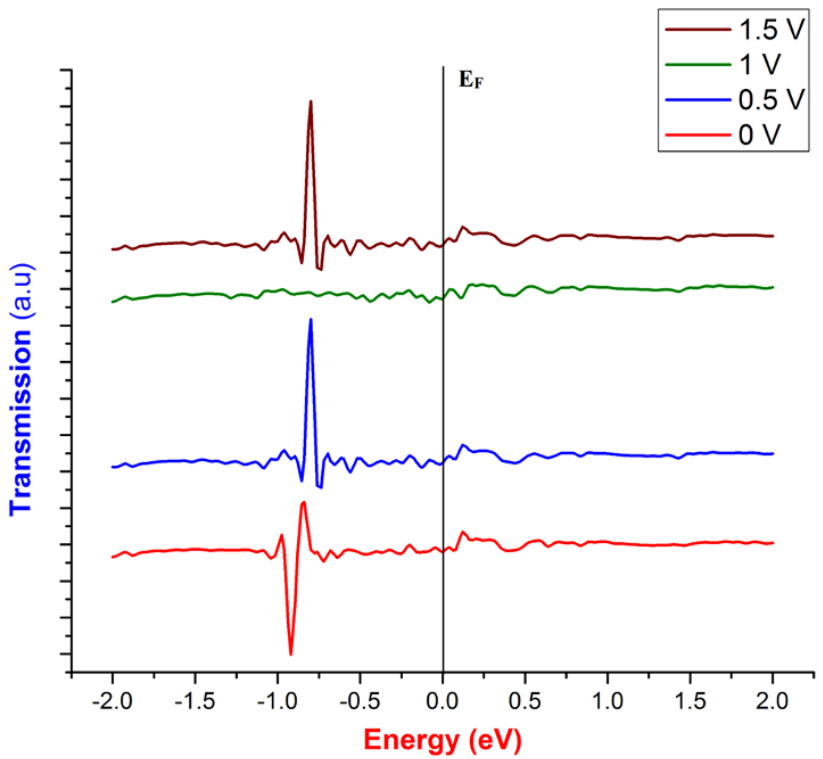

Fig. 7 Transmission spectrum of $\mathrm{CeN}$ nanoribbon at $0,0.5,1,1.5 \mathrm{~V}$ bias

Fig. 8 a Transmission pathways of CeN nanoribbon at 0 V. b Transmission pathways of $\mathrm{CeN}$ nanoribbon at $0.5 \mathrm{~V}$. c Transmission pathways of $\mathrm{CeN}$ nanoribbon at $1 \mathrm{~V}$. d Transmission pathways of $\mathrm{CeN}$ nanoribbon at $1.5 \mathrm{~V}$

the Fermi level in the valence band; on applying a proper bias voltage will move the charge to the conduction band. Figure $3 \mathrm{a}$ and $\mathrm{b}$ represents the PDOS and DDOS spectrum of $\mathrm{CeN}$ nanoribbon at $0.5 \mathrm{~V}$. The bias voltage of $0.5 \mathrm{~V}$ is applied between the electrodes, now in the PDOS spectrum only the contributions of $s, p$ and $d$ orbitals are noticed, there 
(a)

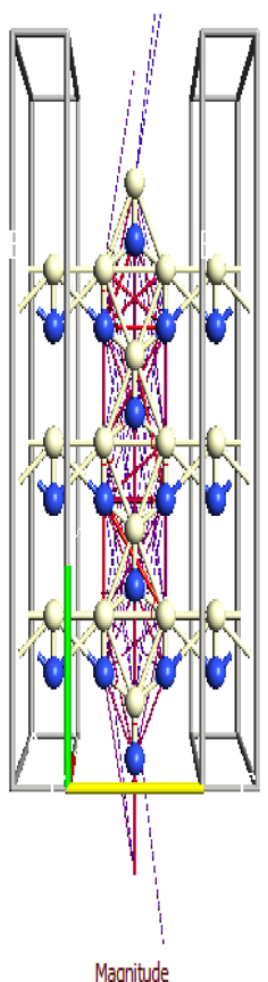

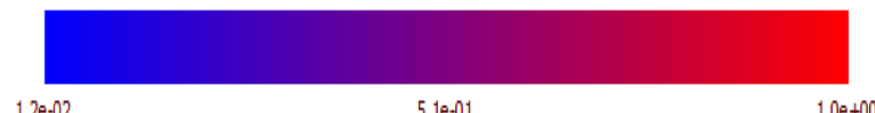

$1.0 e+00 \quad 1.22-02$

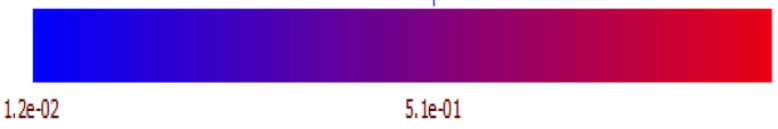

(b)
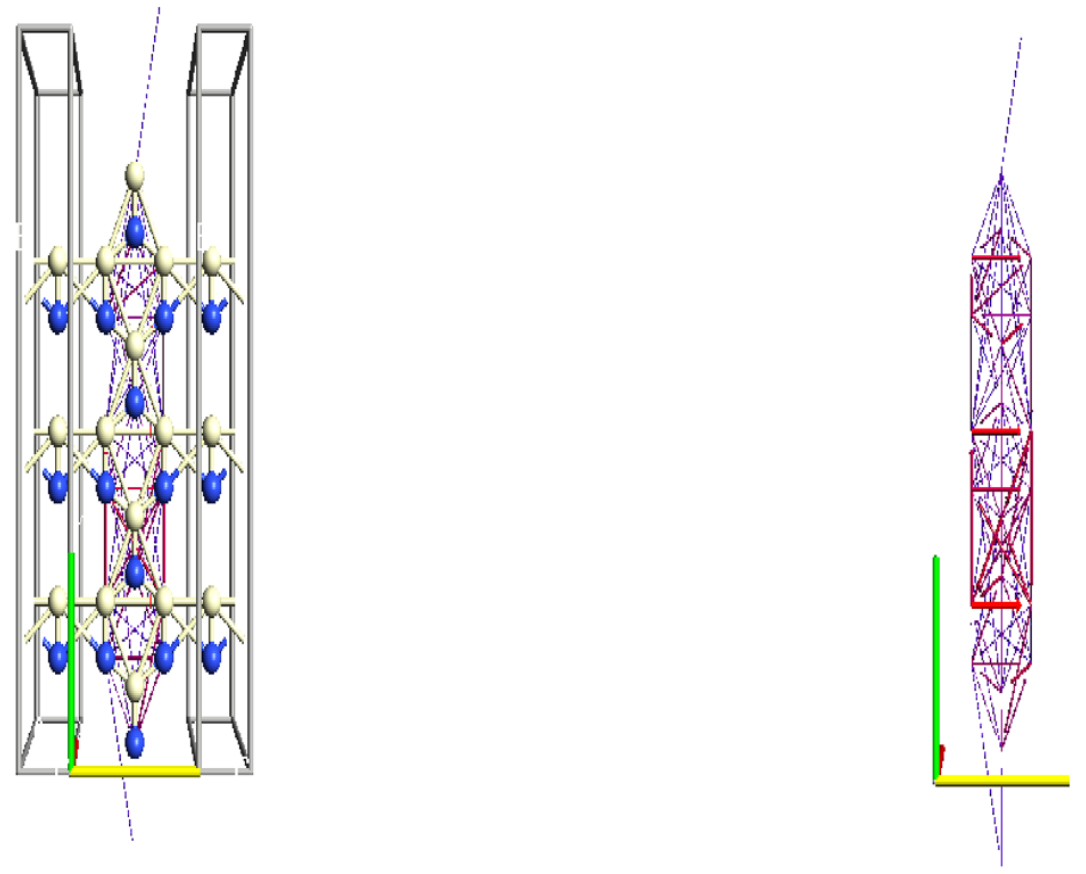

Magnitude
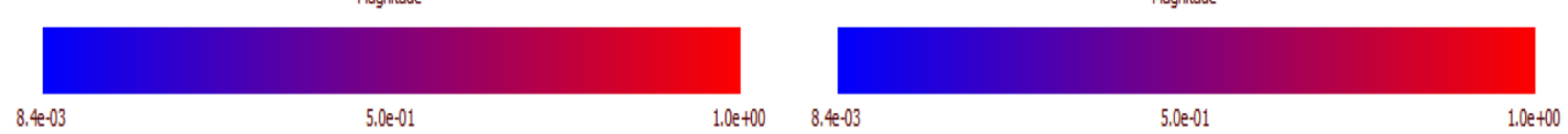
(c)

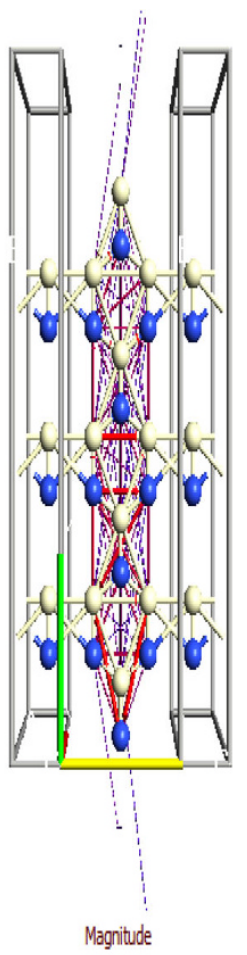

Magnitude

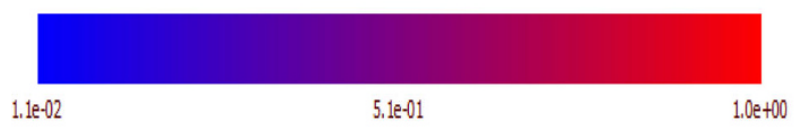

(d)

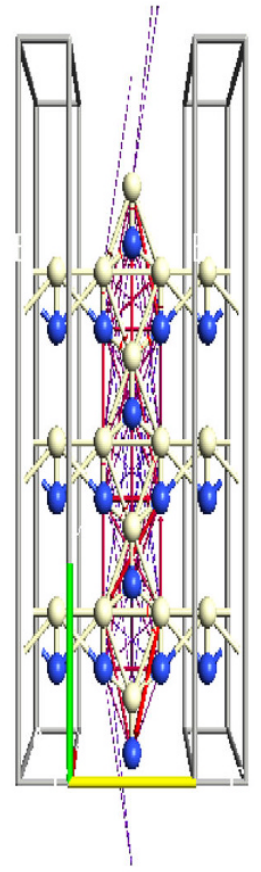

Magnitude

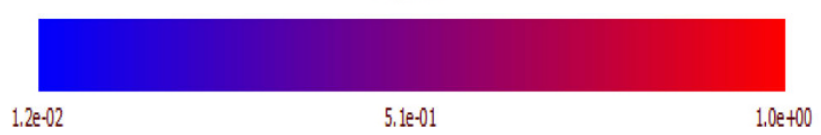

Fig. 8 continued

算 Springer
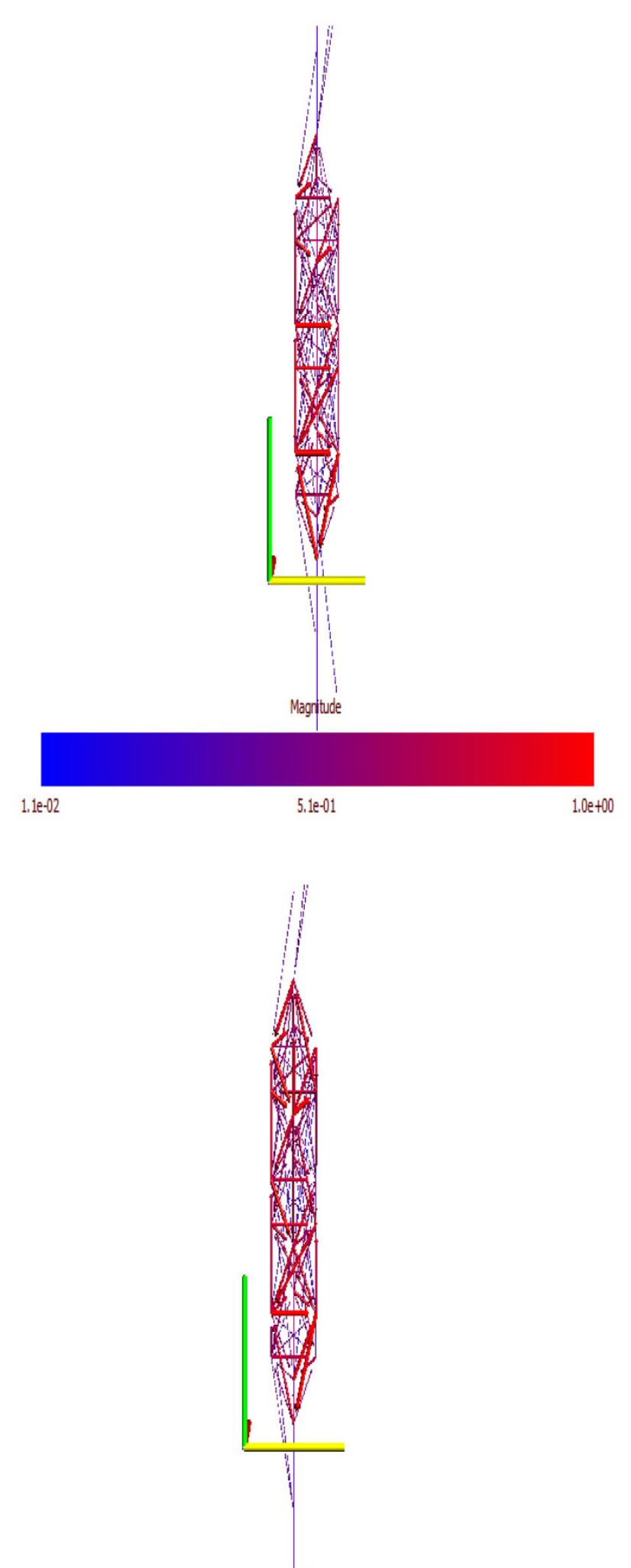

Magritude

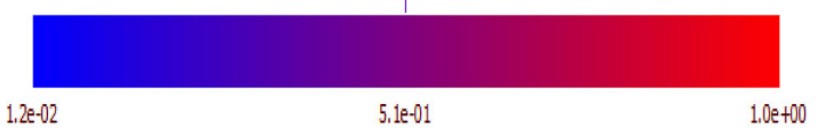


is no contribution of $f$ orbital of cerium atom and the applied bias voltage makes the $f$ orbital electrons to get delocalized. The peak maximum is observed near -0.2 and $-0.8 \mathrm{eV}$. The trend in DDOS spectrum for 0.5 and $0 \mathrm{~V}$ are almost same. Figure $4 \mathrm{a}$ and $\mathrm{b}$ depicts the PDOS and DDOS spectrum of $\mathrm{CeN}$ nanoribbon at $1 \mathrm{~V}$ bias. In the PDOS spectrum of $1 \mathrm{~V}$ bias, only the contribution of $s, p$ and $d$ is seen with more increase in the magnitude at -0.2 and $-0.8 \mathrm{eV}$. The DDOS spectrum gives the same trend with increase in the magnitude, the superposition of the orbitals gives rise to the increase in the magnitude. Figure $5 \mathrm{a}$ and $\mathrm{b}$ represents the PDOS and DDOS at $1.5 \mathrm{~V}$ bias. Interestingly, the same trend is observed in the PDOS spectrum of $1.5 \mathrm{~V}$, there is a consistent peak maximum observed in the valence band of CeN nanoribbon and in DDOS spectrum the same fashion of peaks are observed, this clearly indicates that the geometric structure plays an important role in the peak maximum in the valence band and conduction band of nanoribbon. At zero bias voltage the contribution due to the $f$ orbital is smashed due to the applied bias voltage, the major contribution to the localization of charge arises only because of $s$, $p$ and $d$ orbitals.

Figure 6 depicts the electron density of $\mathrm{CeN}$ nanoribbon. The density of electrons is more in the nitrogen sites than in the cerium sites. The atomic number of nitrogen is 7 , which has the electronic configuration of $1 s^{2} 2 s^{2} 2 p^{3}$; cerium atom has the atomic number of 58 with the electronic configuration of [Xe] $4 f^{1} 5 d^{1} 6 s^{2}$. The overlapping of the orbitals in nanoribbon makes the electrons to accumulate in the nitrogen sites than in cerium atom.

\section{Transport properties of $\mathrm{CeN}$ nanoribbon}

The transmission function of $\mathrm{CeN}$ nanoribbon is represented as $T(E, V)$ for the system which depends on the external bias voltage [22-25]. Equation (1) illustrates the transmission probability as

$T(E, V)=\operatorname{Tr}\left[\Gamma_{\text {up }}(V) G^{\mathrm{R}}(E, V) \Gamma_{\text {down }}(V) G^{\mathrm{A}}(E, V)\right]$

where $G^{\mathrm{R}}$ and $G^{\mathrm{A}}$ is the retarded and advanced Green's function, respectively; $\Gamma_{\text {up, down }}$ are the imaginary parts of the up and down self-energies called as coupling function, respectively. The self-energy depends on the surface Green's function in the electrode regions and arises due to the nearest neighboring interaction between the electrodes and the molecular region. Equation (2) gives the zero bias voltage conductance, which can be found from the transmission probability as

$G=G_{0} T(E, V=0)$

where $G_{0}=2 e^{2} / h$ is the quantum unit of conductance, $h$ is Planck's constant and ' $e$ ' is the electronic charge [26]. When there is no bias, Fermi level of two electrodes is aligned, and transmission of electron from the up to down electrode is equal to the down to up electrode and the net current is zero. When the bias voltage is applied, the chemical potential of the two electrodes no longer remains same and the transmission of charges takes place. The current and conductance are determined by the magnitude of the transmission function between the energy regions.

The peak maximum near the Fermi level $\left(E_{\mathrm{F}}\right)$ contributes to the transport property at the applied low bias voltage [27]. Under zero bias condition, a peak maximum and minimum is observed around $-0.8 \mathrm{eV}$. The localization of charges is seen in the valence band of nanoribbon, there is no peak observed in the conduction band at $0 \mathrm{~V}$ as shown in Fig. 7. A low bias voltage of $0.5 \mathrm{~V}$ makes the charges to get localized at $-0.8 \mathrm{eV}$, and the peak maximum is increased and peak minimum is decreased due to the applied voltage as represented in Fig. 7. A different scenario is observed for $1 \mathrm{~V}$, this voltage causes a perturbation of the charges across the nanoribbon which is seen as a random variation of maxima and minima in both the valence band and conduction band as represented in Fig. 7 . This infers that applied $1 \mathrm{~V}$ is not sufficient to move the charge from the valence band to conduction band. Further increase in the voltage to $1.5 \mathrm{~V}$ causes the localization of the charges in the valence band around $-0.8 \mathrm{eV}$, as shown in the Fig. 7. From the transmission spectrum, it is inferred that for different bias voltage, the localization of the charge changes. The $\mathrm{CeN}$ nanoribbon geometry and the applied bias voltage both influence the transport property of $\mathrm{CeN}$ nanoribbon. The transmission of charges with the bias window depends on the orbital overlapping between cerium and nitrogen atoms in the nanoribbon.

The transmission pathways for $\mathrm{CeN}$ nanoribbon are depicted in Fig. 8a-d. In the case of zero bias, the transmission of electrons is only due to the variation in the chemical potential between the electrodes. The transmission of electrons takes place from up electrode to down electrode (red bold lines) and it gets scattered along the scattering region. When the bias voltage is increased, apart from the chemical potential the bias voltage also causes the transmission along the $\mathrm{CeN}$ nanoribbon. On increasing, the bias voltage further causes the charges to get scattered along the nanoribbon which increases the transmission from up electrode to down electrode. From the observation, it is to be emphasized that increase in the voltage causes the change in the chemical potential across the electrode, which has its impact in the transmission along the nanoribbon.

\section{Conclusions}

The electronic transport properties of $\mathrm{CeN}$ nanoribbon are investigated under different bias voltages using 
density functional theory approach. The orbital of cerium and nitrogen atoms contributes to PDOS spectrum, at zero bias condition the contribution of $f$ orbital is seen in the cerium atom whereas on increasing the bias voltage, the $f$ orbital electrons gets perturbed and there is no contribution of $f$ orbital in other bias voltages. From the result it is inferred that $f$ orbital plays an important role in the transport property of $\mathrm{CeN}$ nanoribbon. The superposition of PDOS gives rise to DDOS spectrum, the localization of charges is seen in the valence band of $\mathrm{CeN}$ nanoribbon. The densities of electrons are seen more in nitrogen sites than in cerium sites. The transmission spectrum of $\mathrm{CeN}$ nanoribbon gives the information about transmission of charges under various bias voltages. The transmission of charges depends on the geometry of the nanoribbon and also due to the overlapping of orbitals between cerium and nitrogen atom. The transmission pathway gets modified due to the applied bias voltage. The information provided in the present work will give an insight to fine-tune $\mathrm{CeN}$ nanostructures with enhanced transport property in nanomaterials.

Open Access This article is distributed under the terms of the Creative Commons Attribution License which permits any use, distribution, and reproduction in any medium, provided the original author(s) and the source are credited.

\section{References}

1. Aerts, C.M., Strange, P., Horne, M., Temmerman, W.M., Szotek, Z., Svane, A.: Half-metallic to insulating behavior of rare-earth nitrides. Phys. Rev. B. 69, 045115-045120 (2004)

2. Svaney, A., Szotekz, Z., Temmermanz, W.M., Lægsgaardy, J., Winter, H.: Electronic structure of cerium monopnictides under pressure. J. Phys. Condens. Matter 10, 5309-5325 (1998)

3. Xiao, S.Q., Takai, O.: Formation and evaluation of $\mathrm{CeN}$ thin films. Thin Solid Films 317, 137-139 (1998)

4. Olsen, J.S., Jørgensen, J.E., Gerward, L., Vaitheeswaran, G., Kanchana, V., Svane, A.: Compressibility and structural stability of $\mathrm{CeN}$ from experiment and theory. The B1-B2 transition. J. Alloys Compd. 533, 29-32 (2012)

5. Xiao, W., Guo, Q., Wang, E.G.: Thickness dependent valence fluctuation of CeN film. Surf. Sci. 572, 296-300 (2004)

6. Soler, J.M., Artacho, E., Gale, J.D., Garcia, A., Junquera, J., Ordejon, P., Portal, D.S.: The SIESTA method for ab initio orderN materials simulation. J. Phys. Condens. Matter 14, 2745-2779 (2002)

7. Fan, Z.Q., Zhang, Z.H., Ming, Q., Tang, G.P., Chen, K.Q.: Firstprinciples study of repeated current switching in a bimolecular device. Comput. Mater. Sci. 53, 294-297 (2012)

8. Jaiswal, N.K., Srivastava, P.: First principles calculations of cobalt doped zigzag graphene nanoribbons. Solid State Commun. 152, 1489-1492 (2012)

9. Fang, C., Cui, B., Xu, Y., Ji, G., Liu, D., Xie, S.: Electronic transport properties of carbon chains between $\mathrm{Au}$ and $\mathrm{Ag}$ electrodes: a first-principles study. Phys. Lett. A 375, 3618-3623 (2011)

10. Zhao, P., Wang, P.J., Zhang, Z., Liu, D.S.: Electronic transport properties of a molecular switch with carbon nanotube electrodes: a first-principles study. Phys. B 405, 446-450 (2010)

11. Lin, L., Jiang, J., Luo, Y.: Elastic and inelastic electron transport in metal-molecule(s)-metal junctions. Phys. E 47, 167-187 (2013)

12. Perdew, J.P., Burke, K., Wang, Y.: Generalized gradient approximation for the exchange-correlation hole of a manyelectron system. Phys. Rev. B. 54, 16533-16539 (1996)

13. Perdew, J.P., Chevary, J.A., Vosko, S.H., Jackson, K.A., Pederson, M.R., Singh, D.J., Fiolhais, C.: Atoms, molecules, solids, and surfaces: applications of the generalized gradient approximation for exchange and correlation. Phys. Rev. B. 46, 6671-6687 (1992)

14. Amin, B., Nazir, S., Schwingenschlogl, U.: Molecular distortion and charge transfer effects in $\mathrm{ZnPc} / \mathrm{Cu}(111)$. Sci. Rep. 3, 1705-1710 (2013)

15. Sharma, I., Randhawa, D.K.K., Singh, K.L.: Quantum simulation study of DNA nucleotide thymine for use in molecular devices. IOSR J. Electr. Commun. Eng. 1, 01-06 (2012)

16. Pashangpour, M., Ghaffari, V.: Investigation of structural and electronic transport properties of graphene and graphane using maximally localized Wannier functions. J. Theor. Appl. Phys. 7, $1-8(2013)$

17. Okuno, Y., Ozaki, T.: First-principles study of multiterminal quantum interference controlled molecular devices. J. Phys. Chem. C. 117, 100-109 (2013)

18. Yuanhua, Q., Daren, G., Chengbu, L.: DFT study of the conductance of molecular wire: the effect of coupling geometry and intermolecular interaction on the transport properties. Sci. Chin. Ser. B. Chem. 49, 492-498 (2006)

19. Qi, Y.H., Guan, D.R., Liu, C.B.: DFT study of the transport properties of molecular wire at low bias. Chin. J. Chem. 24, 326-330 (2006)

20. Srivastava, A., Tyagi, N.: Structural and electronic properties of $\mathrm{AlX}(\mathrm{X}=\mathrm{P}, \mathrm{As}, \mathrm{Sb})$ nanowires: Ab initio study Mater. Chem. Phys. 137, 103-112 (2012)

21. Kuloglu, A.F., Lisesivdin, B.S., Lisesivdin, S.B., Ozbay, E.: Firstprinciples calculations of Pd-terminated symmetrical armchair graphene nanoribbons. Comput. Mater. Sci. 68, 18-22 (2013)

22. Zhang, Y., Wang, F.C., Zhao, Y.P.: Negative differential resistance behavior of silicon monatomic chain encapsulated in carbon nanotubes. Comput. Mater. Sci. 62, 87-92 (2012)

23. Zhao, J.F., Yang, C.L., Wang, M.S., Ma, J.: First-principles analysis of the effect of contact sites on electronic transport properties of diaminofluorene. Phys. B 417, 70-74 (2013)

24. Zhang, Z.H., Guo, C., Kwong, G., Deng, X.Q.: Electronic transport of nitrogen-capped monoatomic carbon wires between lithium electrodes. Carbon 51, 313-321 (2013)

25. Zhang, D., Xu, Y., Zhang, J., Miao, X.: First-principles study of the electronic transport properties in $(\mathrm{GaAs})_{\mathrm{n}}(\mathrm{n}=2-4)$ nanocluster-based molecular junctions. Phys. Lett. A 376, 3272-3276 (2012)

26. Xia, C.J., Liu, D.S., Fang, C.F., Zhao, P.: The I-V characteristics of the butadienimine-based optical molecular switch: an Ab initio study. Phys. E 42, 1763-1768 (2010)

27. Zhao, P., Liu, D.S.: Negative differential resistance behavior in molecular devices based on carbon nanotubes: effects of chirality and electrode-electrode distance. Phys. E. 47, 224-228 (2013) 